\title{
Disrupção por um bilhão: o caso Dollar Shave Club e as implicações das startups em inteligência competitiva
}

Artur Tavares Vilas Boas Ribeiro $^{1}$

${ }^{1}$ Universidade de São Paulo 


\section{Disrupção por um bilhão: o caso Dollar Shave Club e as implicações das startups em inteligência competitiva}

\section{Resumo}

O presente artigo tem como objetivo apresentar impactos do fenômeno das startups disruptivas no contexto da estratégia empresarial e inteligência competitiva. É apresentado um estudo de caso da startup Dollar Shave Club e sua história de disrupção do modelo de lâminas de barbear até a aquisição pela Unilever por um bilhão de dólares. $O$ estudo buscou, por meio da técnica de análise documental, levantar indícios do impacto gerado pela startup considerando tanto a trajetória da pequena empresa quanto os sinais emitidos pela Unilever referentes a um interesse em investimentos e aquisições neste cenário. Como resultados pode-se encontrar uma análise longitudinal com eventos ao longo do tempo que demonstram negligência por parte da Procter \& Gamble quanto às duas situações (emergência da startup e movimentação da Unilever). As conclusões giram em torno do impacto do novo fenômeno na estratégia das empresas e novas possibilidades de inteligência competitiva no mapeamento de ameaças.

Palavras-chave: Startups. Empreendedorismo. Inovação Disruptiva. Inteligência Competitiva.

\section{Introdução}

No começo de 2012, uma pequena startup causava barulho com uma nova proposta na oferta de lâminas de barbear a partir de uma plataforma 100\% digital. Nos primeiros anos de operação da startup, a dominante do mercado preferiu não se movimentar - e, após 4 anos de existência, a startup era comprada pela sua maior rival em uma aquisição de um bilhão de dólares envolvendo uma notável fatia de market share. $\mathrm{O}$ fenômeno das startups causando grandes mudanças está cada vez mais presente, e é sobre isso que o presente artigo irá tratar.

Com a evolução das tecnologias de comunicação, novas possibilidades têm surgido para a criação de negócios e diversos modelos empresariais inovadores surgem em respostas a problemas não antes resolvidos, ou pelo menos não resolvidos de maneira eficiente. $\mathrm{O}$ desenvolvimento de estruturas modernas para criação de softwares, como linguagens de programação mais acessíveis e repositórios open source que facilitam a construção de produtos digitais, fez com que os custos para o desenvolvimento de uma solução caíssem para níveis muito baixos, além de aumentar drasticamente a velocidade da execução (Miller \& Bound, 
2011). Com isso, tem-se um fenômeno em que "cada grande problema no mundo tenha cerca de centenas de times independentes trabalhando para resolvê-lo" (Chu, 2015, p.1. Tradução nossa). Em consequência, a ascensão das pequenas empresas de tecnologia reflete no cenário das grandes empresas, que passam a encontrar uma realidade competitiva mais acirrada capaz de mudar os rumos de estruturas econômicas vigentes (Christensen, Raynor \& McDonald, 2015).

O contexto apresentado, ainda que catalisado pelas mudanças trazidas com um novo mundo globalizado e digitalizado, também encontra raízes em trabalhos clássicos. Destes estudos, destacam-se os de Schumpeter (1942) sobre destruição criativa e os de Ansoff (1975) sobre surpresas estratégicas. Ansoff, por exemplo, destacava a necessidade de firmas estabelecidas estarem atentas a potenciais emergentes com elevada antecedência, de modo a reagir a pequenos sinais de movimentação competitiva antes que as transformações emergentes já tivessem força o suficiente a ponto de impedir uma reação a tempo por parte das incumbentes. $\mathrm{O}$ aspecto central da negligência estratégica avançou em trabalhos do professor Clayton Christensen, que estudou novos fenômenos de movimentação de inovadores, tema sob o qual cunhou o termo 'inovação disruptiva' (Christensen, 1992; Bower \& Christensen, 1995).

Embora os estudos ligados à inovação tenham se aprofundado nas mudanças competitivas em um cenário de negligência e surpresas estratégicas, pouco tem se falado sobre os desdobramentos nos estudos sobre inteligência competitiva frente a emergência de pequenas empresas inovadoras. Dado que o processo de inteligência competitiva é um processo de construção de mecanismos que garantem informação acionável acerca de movimentações da competição (Prescott, 1995), o aumento dos casos envolvendo surpresas estratégicas com as startups aponta para a existência de um gap na teoria. Esse gap apresentou-se como uma oportunidade de estudo exploratório que norteou a pesquisa a partir da seguinte questão: quais as implicações do fenômeno das startups nas estratégias de inteligência competitiva das empresas estabelecidas?

Definida a motivação, o estudo se estruturou em torno de um caso recente de movimentação competitiva envolvendo uma startup gerando uma disrupção no mercado de lâminas de barbear: o caso da Dollar Shave Club. Com um estudo de caso, o presente estudo tem como objetivo analisar as implicações nas práticas de inteligência competitiva dado o contexto da emergência de pequenas empresas de tecnologia com modelos disruptivos. Para o cumprimento deste objetivo, as próximas sessões do artigo se desdobrarão (i) no referencial teórico - analisando os tópicos relacionados a estratégia empresarial, competição e inovação disruptiva; (ii) na apresentação detalhada do método científico utilizado; (iii) no estudo do caso - com a apresentação do fenômeno e um detalhamento do contexto de sinais fracos identificados; (iv) nas discussões; e, por fim, (iv) na conclusão do artigo.

\section{Referencial Teórico}




\subsection{Estratégia empresarial e inteligência competitiva}

Com o amadurecimento das práticas de gestão empresarial consequentes do amadurecimento dos modelos econômicos, tem-se também o desenvolvimento das escolas de administração. No desenvolver das bases clássicas para a administração de empresas, as discussões sobre estratégia no campo empresarial ganharam força a partir de suas primeiras aplicações em empresas como General Motors, na primeira metade do século XX, refletindo em consequentes registros e sistematizações, tal como no livro "My years with General Motors" (Sloan, 1964). Desse movimento originaram-se os primeiros trabalhos acadêmicos sobre estratégia empresarial de Ansoff (1965) e seus estudos envolvendo a atuação racional em cenários competitivos incertos, como o artigo "Managing strategic surprise by response to weak signals" (Ansoff, 1975). Neste estudo, Ansoff traz um conceito a partir de um paralelo com cenários de guerra: o conceito de surpresas estratégicas.

As surpresas estratégias são eventos de alto impacto que surpreendem organizações e gestores, encontrando os mesmos despreparados mesmo quando se possuía acesso a informações suficientes para uma prevenção. Para o autor, embora haja sistemas e modelos de previsão, "quando uma surpresa potencial se origina em uma tecnologia alienígena, com um concorrente anteriormente desconhecido, com uma nova coalizão política ou com um novo fenômeno econômico, a simples extrapolação não é suficiente" (Ansoff, 1975, p. 22), indicando a necessidade de uma melhor organização para identificação de potenciais ameaças, bem como um preparo mais preciso para eventuais necessidades de ação. Segundo Ansoff, o fenômeno de ameaças emergentes tornava-se mais complexo devido ao paradoxo entre a natureza da informação e o cenário de uma possível ação reativa. Neste paradoxo, esperar até um nível de informação madura o suficiente poderia fazer com que, ao decidir agir, a organização se encontrasse atrasada demais para ser efetiva; ao passo que decidir atuar com informações vagas demais poderia levar à falta de especificidade incapaz de nutrir uma boa estratégia.

Diante dos desafios apresentados, diversas práticas para a análise do ambiente externo e atenção a potenciais surpresas competitivas foram desenvolvidas. Ansoff se concentrou na atenção à evolução de sinais fracos que poderiam representar uma futura movimentação competitiva, propondo uma reação gradual através da amplificação e resposta a sinais fracos. Outros autores também propuseram seus mecanismos para lidar com essas incertezas, como ferramentas de business intelligence (Blanco \& Lesca, 1998) e escaneamento ambiental (Choo, 2001). Desta conjuntura nasceu o conceito de Inteligência Competitiva, uma disciplina voltada ao entendimento do cenário competitivo de modo a gerar informações acionáveis para suporte ao processo de tomada de decisão (Sharp, 2009). Com o avanço das relações entre desenvolvimento tecnológico e competitividade nas organizações, a inteligência competitiva encontrou suas especializações, como o conceito de inteligência tecnológica:

"The goal of technology intelligence is to exploit potential opportunities and to defend against potential threats, through prompt delivery of relevant information about technological trends in the environment of the company. Technology intelligence encompasses the activities related to the collection, analysis and communication of relevant information on technological trends to support

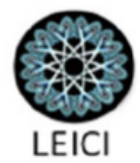


technological and more general decisions of the company. According to this definition, technology intelligence includes the monitoring and analysis of individual competitors as well as universities and start-up companies. ”(Lichtenthaler, 2003, p.

O cenário da internet sendo caminho para pequenas empresas emergentes e disruptivas também é apresentado como um elemento complementar à inteligência competitiva (Teo e Choo, 2001), podendo se mostrar útil na coleta de novas informações e identificação de potenciais emergentes. Para o presente estudo, será observado o papel deste novo cenário na apresentação de sinais fracos e as principais implicações para inteligência competitiva.

\subsection{As pequenas empresas emergentes e a disrupção}

Os debates sobre forças inovadoras destrutivas têm pilares nos estudos de Schumpeter com o conceito de destruição criativa: uma transformação de cenários que "revoluciona incessantemente uma estrutura econômica a partir dela mesma, destruindo incessantemente a estrutura antiga, criando incessantemente uma nova estrutura" (Schumpeter, 1942, p.83). A evolução dos conceitos e a ascensão do conceito de 'inovação disruptiva' é apresentada pela seguinte linha temporal de Yu \& Hang (2010):

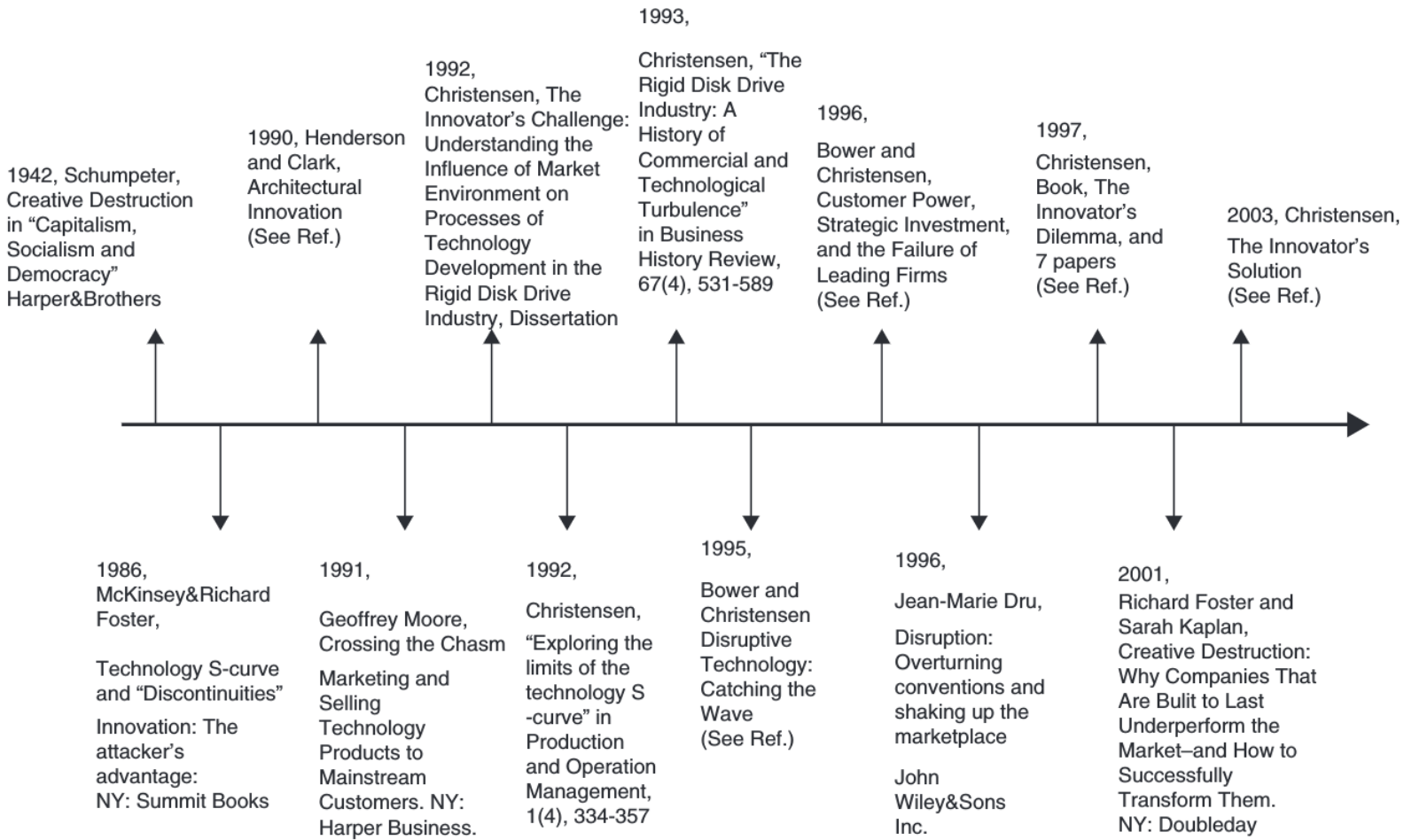

Figura 1: Linha do tempo da evolução da teoria de inovação disruptiva (Yu \& Hang, 2010).

Embora muito disseminada, a teoria da inovação disruptiva frequentemente é má interpretada. Muitos se apropriam do termo utilizando uma definição que é mais apropriada ao conceito de inovação destrutiva: uma nova tecnologia superior e mais barata que faz com que 
os modelos anteriores percam seu valor e sejam descontinuados (Yu \& Hang, 2010). No caso da inovação disruptiva, ressalta-se que a tese central está no processo de sua ocorrência: uma inovação que inicialmente se concentrava em mercados menos atrativos, com características secundárias superiores, mas sendo inferior na característica primária para o mercado (como, por exemplo, tecnologia), que, ao se desenvolver e amadurecer, atinge as expectativas dos mercados maiores e substitui os grandes players pelos outros elementos muito superiores. $\mathrm{O}$ ponto central da teoria proposta por Bower \& Christensen (1995) é o fato de, ao apostar mercados menores e utilizando-se de uma característica central inferior, os entrantes disruptivos são negligenciados nas análises competitivas dos grandes players - o que faz com que tais entrantes se tornem uma surpresa estratégica, nos termos de Ansoff (1975), após se tornarem competitivos nas características primárias.

Exemplificando em situações contemporâneas, Christensen, Raynor \& McDonald (2015) trazem o exemplo da empresa Netflix como um entrante que passou pelo processo de disrupção: inicialmente a empresa não possuía grandes lançamentos e seus filmes, enviados por correio, demoravam para chegar (característica primária inferior). Apesar disso, a comodidade da distribuição e a interface online (características secundárias) se mostravam interessantes para uma pequena parcela de clientes - um contexto não atrativo para a Blockbuster, fazendo com que o potencial dessa nova estrutura fosse negligenciada em estratégias competitivas. Com o desenvolvimento das novas tecnologias que permitiram distribuição instantânea e em boa qualidade, além da evolução da Netflix na oferta de filmes e séries reconhecidas, deu-se a situação de disrupção da Blockbuster e do modelo de negócios de locadoras no mundo inteiro. O conceito central da teoria da inovação disruptiva é, portanto, esta miopia dos grandes players ao negligenciar entrantes que se concentram em mercados menores, com soluções primárias inferiores, mas que possuam elementos complementares e potencial de desenvolvimento de soluções em uma evolução destrutiva. Para o autor, tal compreensão é fundamental para evitar que sua teoria se torne vítima do próprio sucesso:

\begin{abstract}
"In our experience, too many people who speak of "disruption" have not read a serious book or article on the subject. Too frequently, they use the term loosely to invoke the concept of innovation in support of whatever it is they wish to do. Many researchers, writers, and consultants use "disruptive innovation" to describe any situation in which an industry is shaken up and previously successful incumbents stumble. But that's much too broad a usage. The problem with conflating a disruptive innovation with any breakthrough that changes an industry's competitive patterns is that different types of innovation require different strategic approaches.

(...) If we get sloppy with our labels or fail to integrate insights from subsequent research and experience into the original theory, then managers may end up using the wrong tools for their context, reducing their chances of success. Over time, the theory's usefulness will be undermined."

(Christensen, Raynor \& McDonald, 2015, p.4)
\end{abstract}

Realizando uma revisão da literatura sobre a inovação disruptiva, Yu \& Hang (2010) trazem os principais pontos identificados para grandes empresas serem disrompidas, partindo da premissa que "a maioria dos problemas reside mais nas empresas estabelecidas em si que em seu ambiente e contexto" (p. 441): 
(i) Recursos humanos: gestores com um olhar muito viciado baseado em uma longa experiência na grande corporação, ou concentrados demais em investir em seus feudos. Aqui também se encontram os modelos de recompensa, punições e bonificações, que usualmente minimizam a disposição dos colaboradores de assumir riscos maiores.

(ii) Cultura organizacional: padrões de ação que retroalimentam uma inércia corporativa;

(iii) Alocação de recursos: estrutura das rotinas dos recursos - geralmente priorizando atividades em projetos existentes e tratando potenciais inovações internas como secundários.

(iv) Estrutura organizacional: estrutura de fornecedores, complexidade organizacional e cadeia de distribuição engessada o suficiente para impedir mudanças ou inovações.

Tais elementos corroboram com o que Christensen \& Bower (1996) diagnosticam ao afirmar que, após situações de impossibilidade de explorar mercados disruptivos em grandes empresas, "novas companhias, geralmente compostas por membros frustrados de times de engenharia de firmas estabelecidas, eram constituídas para explorar novas arquiteturas de produtos disruptivas" (p. 209).

Assim como houve desdobramentos a partir dos estudos de Ansoff (1975) em torno de mecanismos para melhor se preparar para supresas utilizando sinais fracos, as pesquisas sobre inovação disruptiva abriram um campo de estudo sobre melhores práticas para se evitar disrupções vindas da competição. Yu \& Hang (2010) identificaram na literatura pontos como: (i) times separados, às vezes geograficamente distantes (modelo conhecido como skunkworks), para exploração de projetos inovadores; (ii) políticas de incentivo de longo prazo com análises subjetivas, em vez de baseadas em fórmulas com métricas pré-determinadas; (iii) unidades menores de negócio, com práticas de gestão mais capilarizadas que valorizem a incerteza de projetos nascentes; (iv) colaboração com startups e estratégias de aquisição de propriedade intelectual externa; (v) realização de conjoint analysis para identificar, a partir de curvas de atributos, potenciais disrupções.

Deste modo, o arcabouço teórico de inteligência competitiva e de inovação disruptiva se encontram na necessidade de se atentar de maneira profissional aos movimentos dos pequenos emergentes. Dentro do contexto de startups, que possuem grande velocidade em sua disseminação e modernização de modelos, esta competência se torna fundamental para organizações de todos os tamanhos e setores. 


\section{Instrumentos e método}

Partindo da motivação inicial do presente estudo - a análise das implicações do novo contexto de startups disruptivas nas práticas de inteligência competitiva -, foi pensado como método o Estudo de Caso. A escolha se deu a partir da observação de um fenômeno no campo da estratégia empresarial: o caso da startup Dollar Shave Club e sua disrupção no mercado de lâminas de barbear. Deste modo, o estudo de caso se mostrou relevante para análise criteriosa sobre um fenômeno único capaz de trazer à tona padrões relacionados à fronteira da pesquisa em inteligência competitiva e inovação (Yin, 2013).

Sendo o Caso Dollar Shave Club um interessante caso de surpresa estratégica a ser analisado para identificar as implicações citadas acima, o mecanismo de coleta de dados foi realizado por meio de levantamento de notícias, artigos e discursos disponíveis publicamente para analisar potenciais sinais de mudança competitiva e o processo de ruptura gerado pela startup. Assim sendo, o presente artigo se trata de um estudo exploratório baseado em uma pesquisa documental baseado nas orientações metodológicas de (Gil, 2008). Segundo o autor, esse tipo de estudo se mostra relevante por possibilitar uma leitura do passado que consiga fazer emergir evidências de processos de mudança social e cultural. Dado que este estudo se orienta às possíveis implicações do novo fenômeno das startups em mudanças gerenciais, identificouse no procedimento um caminho sustentável. Continuando nas bases do autor, a análise dos dados contou com com fundamentos de Bardin (1977) e Berelson (1952).

De modo a tornar a pesquisa mais consistente, foi adotado um protocolo que busca cumprir as propostas de Gil (2008, p. 147) que busca valer-se "dos registros cursivos, que são persistentes e continuados". Foram utilizados os meios digitais para alinhar-se com o tema estudado e as buscas se orientaram a palavras-chave 'startups', 'inovação', 'empreendedorismo', 'novos negócios', 'tecnologia', 'pequenas empresas'. O objetivo dessa busca, realizado no site institucional de duas grandes empresas - Unilever e Procter \& Gamble -, era verificar e comparar a postura das duas em relação ao fenômeno por meio de discursos institucionais, notícias ou decisões estratégicas comunicadas. Também foram realizadas buscas em buscadores abertos com o objetivo de encontrar notícias envolvendo as marcas e o fenômeno das startups e de modelos inovadores emergentes. A partir disso, os registros foram organizados e parametrizados em uma linha do tempo para analisar um paralelo com o surgimento e ascensão da Dollar Shave Club.

A apresentação do caso buscou seguir a proposta da generalização naturalística de Stake (1978), cuja riqueza descritiva compõe o elemento central para que o leitor navegue pelo conteúdo de modo a construir seus aprendizados. Como reforçado por Alves-Mazotti (2006, p.648), "por meio de uma narrativa densa e viva, o pesquisador pode oferecer oportunidade para a experiência vicária, isto é, pode levar os leitores a associarem o que foi observado naquele caso a acontecimentos vividos por eles próprios em outros contextos". Deste processo 
de associação espera-se que gestores de empresas sejam capazes de melhor se organizar competitivamente diante do contexto da emergência de startups.

\section{$4 O$ estudo de caso}

\subsection{O caso Dollar Shave Club}

Fundada em 2012, a abordagem estratégica da startup Dollar Shave Club se sustentou em redução de custos a partir de um redesenho do modelo de negócio em vendas de lâminas de barbear. No modelo vigente, as empresas produzem os aparelhos, distribuem em pontos de vendas diversos (supermercados, drogarias, mercados locais e outros), realizam ações de promoção em diversas mídias (televisão, revistas, outdoors e outros), trazendo estruturas de custo elevadas por alguns fatores:

(i) Patrocínios de classe mundial: Liga Mundial de Baseball, Equipe Inglesa de Rugby, Roger Federer, Tiger Woods e outros;

(ii) Custos de distribuição em pontos de venda físicos, além dos custos envolvidos com embalagem capaz de reduzir casos de furto;

(iii) Constante alteração do produto para renovação de mercados, trazendo aparelhos de barbear com maior número de lâminas e outras inovações incrementais.

A proposta da startup se concentrou em reduzir as estruturas de custos a partir de um serviço de assinaturas online cujo principal produto é um kit de barbear de baixo custo (com apenas duas lâminas na sua versão mais famosa) entregue frequentemente na casa do cliente com preços bastante reduzidos ao da competição tradicional. Além da proposta de valor reduzir o preço final, ela agrega na comodidade de receber as lâminas em casa e se comunica com o cliente de maneira inovadora, com um serviço de atendimento e personalização nos envios que tornou a startup reconhecida pela construção da marca, formato de comunicação inteligente e da lealdade construída com seus clientes. Os diferenciais eram reforçados por campanhas de marketing provocativas (usualmente com bastante humor e ironias em relação aos modelos tradicionais de lâminas de barbear) de baixo custo - em plataformas gratuitas como youtube e redes sociais. O lançamento da empresa, por exemplo, foi considerado um caso de sucesso por ter alcançado 4 milhões de visualizações na plataforma Youtube na primeira semana, como apresentado no artigo "Big Brands Should Fear The 'Dollar Shave Club' Effect" (Vinjamuri, 2012), escrito em 2012 pela Forbes:

"The \$13 billion shaving industry is in a lather this week as the start-up DollarShaveClub.com is bursting through to the mainstream media with a cheeky attitude and a viral video with over 4 million views. Why has Dollar Shave Club broken through where others have not? For one thing, the company understands social media, and its YouTube video does a great job of skewering the shaving giants." (Vinjamuri, 2012) 
Em paralelo ao crescimento da startup, as duas gigantes do mercado de bens de consumo - Unilever e Procter \& Gamble (P\&G) - adotaram estratégias diferentes no monitoramento e relacionamento com empresas emergentes. Sem grandes ações reconhecidas no ambiente de startups, a P\&G reagiu aos movimentos da Dollar Shave Club entrando no mercado de assinaturas online apenas após dois anos, em 2014, com o "Gillete Shave Club". Posteriormente, a empresa abriu um processo contra a Dollar Shave Club, em dezembro de 2015, alegando questões relacionadas a uma patente da lâmina de barbear - movimento que levantou discursos acerca de uma postura anticompetitiva por parte da $\mathrm{P} \& \mathrm{G}$. Na contramão das posturas adotadas pela rival, a Unilever já demonstrava interesse em um relacionamento saudável com startups que poderiam causar disrupção em seus mercados, desenvolvendo braços de investimentos, programas de apoio a empresas nascentes e ações de relacionamento com potenciais emergentes. Nas estratégias da Unilever, tem-se:

-Unilever Ventures: o braço de Venture Capital e Private Equity da empresa. Tem como foco investir em empresas nascentes nos mercados digitais e de cuidados pessoais, oferecendo acesso ao ecossistema Unilever, seus ativos e expertises.

-Unilever Foundry: programa de apoio a startups criado em 2014 que oferece suporte para o desenvolvimento de startups com potencial de virem a ser parceiras da empresa. O suporte conta com mentorias e direcionamento para que as startups possam vir a ser contratadas em testes-piloto oferecendo serviços para organizações da Unilever, um processo no qual há o refinamento das soluções e amadurecimento das empresas. Além de ter a oportunidade de se tornar um fornecedor para a Unilever, o programa é ligado ao Unilever Ventures, permitindo que startups possam vir a ser investidas pela empresa. -Eventos pontuais: eventos de ativação e relacionamento com empreendedores, como a premiação "Unilever Sustainable Living Young Entrepreneurs Awards", que apoia projetos de impacto de empreendedores em países emergentes, reconhecido mercado da empresa.

Com o lançamento da Unilever Foundry, em 2014, o discurso da companhia se intensificou quanto à necessidade de atenção às startups que poderiam gerar grandes mudanças competitivas no contexto da empresa. Em Julho de 2016, a empresa anunciava sua aquisição da Dollar Shave Club pela quantia de um bilhão de dólares em dinheiro, representando uma das maiores movimentações do ano no cenário de startups e a entrada da Unilever em um dos principais mercados da rival $\mathrm{P} \& \mathrm{G}$.

\subsection{Sistematização de dados}

No caso em questão, é percebida uma "surpresa estratégica" no cenário competitivo da Procter \& Gamble, surpresa precedida por diversos sinais que poderiam indicar alguma movimentação a ser realizada pela Unilever. Para fins de aprofundamento, foram elencados dois grandes blocos de sinais fracos acerca dessa movimentação competitiva: (i) eventos no mercado de lâminas de barbear; (ii) discursos públicos de executivos Unilever sobre o cenário

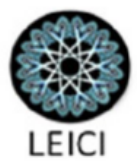


de startups. Acredita-se que a organização visual dos dois blocos permite melhor compreensão dos sinais e facilita a leitura das decisões das duas corporações em relação a um competidor emergente.

\begin{tabular}{|c|c|}
\hline Data & Eventos relevantes na relação Dollar Shave Club e P\&G \\
\hline $03 / 2012$ & Dollar Shave Club levanta US\$ 1 milhão com Kleiner Perkins e outros \\
\hline $03 / 2012$ & $\begin{array}{l}\text { Dollar Shave Club viraliza com vídeo-propaganda no Youtube atingindo mais de } 4 \text { milhões de } \\
\text { visualizações em poucos dias. }\end{array}$ \\
\hline $04 / 2012$ & $\begin{array}{l}\text { "Big Brands Should Fear The 'Dollar Shave Club' Effect", reportagem da Forbes aponta os } \\
\text { riscos de startups emergentes destruírem mercados das grandes empresas de bens de consumo. }\end{array}$ \\
\hline $11 / 2012$ & Dollar Shave Club levanta US\$ 9,8 milhões. \\
\hline $06 / 2013$ & $\begin{array}{l}\text { Dollar Shave Club chama atenção na internet após ação de dia dos pais em rádios locais } \\
\text { americanas. }\end{array}$ \\
\hline $06 / 2013$ & Dollar Shave club se torna referência em marketing com mais de 330.000 assinantes mensais. \\
\hline $10 / 2013$ & Dollar Shave Club levanta US\$ 12 milhões. \\
\hline $04 / 2014$ & Procter and Gamble entra no mercado de assinaturas com Gillete Shave Club. \\
\hline $06 / 2014$ & Dollar Shave Club é reconhecida como história de sucesso em construção de marca. \\
\hline $09 / 2014$ & $\begin{array}{l}\text { Dollar Shave Club é reconhecida pela excelência em gestão de mídias sociais, com cerca de } 1 \\
\text { milhão de seguidores no facebook e atuação em diversos meios (twitter, youtube, vine etc.). }\end{array}$ \\
\hline $09 / 2014$ & Dollar Shave Club levanta US\$ 50 milhões. \\
\hline $11 / 2014$ & Dollar Shave Club aposta em comerciais de televisão e vira sensação na internet. \\
\hline $03 / 2015$ & $\begin{array}{l}\text { Dollar Shave Club reconhecida como case de branding pela Brandfolder Digital Asset } \\
\text { Management. }\end{array}$ \\
\hline $09 / 2015$ & Dollar Shave Club reconhecida pela excelência em Customer Service pelo LA Times. \\
\hline $09 / 2015$ & Participação de mercado online: $21 \%$ P\&G x 54\% Dollar Shave Club. \\
\hline $11 / 2015$ & Dollar Shave Club levanta US\$ 91 milhões em series D. \\
\hline $12 / 2015$ & P\&G abre processo contra Dollar Shave Club alegando ter infringido uma patente. \\
\hline
\end{tabular}

Quadro 1: Eventos relevantes da relação P\&G e Dollar Shave Club (Fonte: dos autores).

Acima, as datas demonstrando o crescimento da Dollar Shave Club e as movimentações da Procter and Gamble em relação à mudança do cenário competitivo. Em paralelo, pode-se 
comparar a postura da Unilever em relação às startups nos discursos apresentados pelos seus executivos. Tais discursos podem ser interpretados como sinais fracos para a aquisição que viria a acontecer em 2016.

\begin{tabular}{|c|c|}
\hline Data & Discursos públicos de executivos Unilever \\
\hline Março, 2014 & $\begin{array}{l}\text { "It is important as marketers to constantly adapt and get ahead of the world we live in. With } \\
\text { technology - and even more so with mobile technology - the pace of change is accelerating } \\
\text { exponentially. Working with young, high-growth companies that are partly inventing the future } \\
\text { will enable us to continue to pioneer new technologies and platforms which can help our brands } \\
\text { engage more meaningfully with people around the world." Marc Mathieu, Senior Vice } \\
\text { President Marketing }\end{array}$ \\
\hline Maio, 2014 & $\begin{array}{l}\text { "We strive to be at the forefront of marketing and media innovation; to have our brands engage } \\
\text { in the most creative, efficient and effective ways with the people we serve. Although we have } \\
\text { been working with start-ups for years, we now want to scale up our efforts and, ultimately, } \\
\text { embed this as a way of working throughout our organisation." Keith Weed, Chief Marketing } \\
\text { and Communications Officer }\end{array}$ \\
\hline Maio, 2014 & $\begin{array}{l}\text { "Through mentoring, we hope to contribute to the entrepreneurial ecosystem and build } \\
\text { businesses who might eventually become our global strategic partners. Mentoring also enables } \\
\text { our marketers across the organisation to engage directly with start-ups and be inspired by new } \\
\text { ideas and ways of working." Luis Di Como, Senior Vice President Media }\end{array}$ \\
\hline Maio, 2014 & $\begin{array}{l}\text { "Through The Unilever Foundry, we hope to make more start-ups aware of this opportunity to } \\
\text { receive investment from the world's second largest advertiser." Olivier Garel, Head of Unilever } \\
\text { Ventures }\end{array}$ \\
\hline Setembro, 2015 & $\begin{array}{l}\text { "I'm not losing share to P\&G and other consumer packaged goods companies, I'm losing it to } \\
\text { small players who are finding niche products." Shawn O'neal, VP-Global People Data and } \\
\text { Marketing Analytics }\end{array}$ \\
\hline Novembro, 2015 & $\begin{array}{l}\text { "If you think about our categories, they're also being disrupted by startups. The Ubers or } \\
\text { Airbnbs of various categories are popping up all over the world. And we think we've got an } \\
\text { opportunity for them to go from startup to scaleup in the same way that we've worked with } \\
\text { digital marketing startups." Jeremy Basset, head of the Unilever Foundry }\end{array}$ \\
\hline Novembro, 2015 & $\begin{array}{l}\text { "The small and fast eat the big and slow, it's been showcased in many industries. So we want to } \\
\text { make sure that we're leading from the front as opposed to just reacting." Babs Rangaiah, VP- } \\
\text { Global Media Innovation and Ventures }\end{array}$ \\
\hline
\end{tabular}

Quadro 2: Sinais fracos presentes nos discursos de executivos Unilever. (Fonte: dos autores)

\section{Discussões}

Ao se analisar os dados levantados em relação ao caso, é possível identificar um fenômeno com duas vertentes de análise de sinais fracos no cenário competitivo do mercado de lâminas de barbear da Procter \& Gamble. O primeiro sendo a emergência de uma startup com um modelo de negócios destrutivo ao modelo da $\mathrm{P} \& \mathrm{G}$, enquanto o segundo as estratégias da 
Unilever para aproximação de startups e entrada em novos mercados a partir de investimentos e aquisições. É possível, com os dados, pontuar uma dupla negligência por parte da P\&G que se desdobrou na surpresa estratégica enfrentada pela empresa. O reflexo se encontrou em números alarmantes para a companhia, como apontado em um relatório de Abril de 2017, que afirma que o negócio de barbear da $P \& G$ foi a única divisão a demonstrar uma queda nas vendas orgânicas, relacionando tal fato com o novo cenário competitivo afetado pelo modelo de distribuição online (Linnane, 2017).

Em paralelo ao referencial teórico, faz-se importante a reflexão na mudança das características dos sinais fracos, que inicialmente se sustentavam em estratégias como análise de patentes, observação de funcionários, diálogo com fornecedores e outros pontos. $\mathrm{O}$ caso da ascensão da Dollar Shave Club apresenta um novo horizonte no contexto de sinais fracos: a atenção em elementos relacionados às mídias sociais e fenômenos de viralização, que podem ser uma fonte para a área de inteligência competitiva das empresas, não somente se limitando às atividades de marketing. $O$ tempo de resposta da $P \& G$, levando dois anos após a viralização do vídeo da startup emergente, aponta relativa negligência ao fenômeno ocorrido em tais canais. Em paralelo, a Unilever se mostrava atenta às startups, com seus executivos não só mapeando o movimento de potenciais inovações disruptivas, mas também investindo, atuando em conjunto e, mais importante, reforçando uma cultura organizacional que reconhecia os perigos das pequenas empresas inovadoras ganhando mercados importantes - nível de preparo notavelmente alinhado às estratégias de proteção contra a disrupção apresentadas por $\mathrm{Yu} e$ Hang (2010).

No que foi tratado como uma "segunda negligência" da Procter \& Gamble, o tempo de resposta entre Março de 2014, quando aconteciam os primeiros discursos da Unilever sobre uma atuação mais intensiva com startups, e Julho de 2016, momento da aquisição da Dollar Shave Club, reforça os postulados de Ansoff (1975) sobre as surpresas estratégicas serem normalmente acompanhadas de sinais fracos que poderiam ter sido tratados previamente de modo a evitar impactos negativos da competição.

Quanto à característica disruptiva da Dollar Shave Club, faz importante o ponderamento baseado no conceito original de Christensen (1992), que defende que a disrupção ocorre a partir da evolução da performance em atributos tradicionais no sentido do que é aceitável pelo mercado. Podemos afirmar que a startup iniciou sua estratégia atacando um mercado menor com um produto de qualidade inferior ao do mercado (barbeador com duas lâminas para adotantes iniciais com disposição à compra online), tudo isso a um valor menor e com características secundárias altamente diferenciadas (relação com o consumidor, branding e estratégias de comunicação que garantem alta fidelidade dos clientes). Sobre a evolução da performance para um padrão aceitável, tem-se dois elementos: o primeiro sendo a ampliação da linha de produtos para modelos com mais lâminas e outras características; o segundo, e mais importante, ações de marketing que fortaleceram o posicionamento da empresa e reduziram a expectativa de performance por parte dos clientes (que antes eram estimulados por melhorias

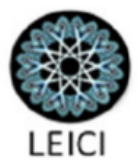


incrementais continuas apontadas como desnecessárias). A evolução dialoga com o proposto por Bower \& Christensen (2015) ao afirmar que os mercados que sofrem disrupção acabavam por ter produtos muito acima das expectativas dos clientes, sendo que os emergentes atingiam o nível do aceitável e superavam com características complementares. No presente caso, as características complementares residiram em uma relação muito forte entre clientes e marca. Deste modo, é possível concluir que o caso da Dollar Shave Club é um caso de inovação disruptiva do mercado de barbear, cabendo reforçar que a inovação se deu muito mais no modelo de negócios da empresa, e não em sua tecnologia.

\section{Considerações finais}

O presente artigo buscou, por meio da utilização do estudo de caso da Dollar Shave Club, chamar a atenção para o fenômeno da inovação disruptiva sob a ótica dos conceitos de sinais fracos propostos por Ansoff (1975). O objetivo da pesquisa - a análise das implicações do novo contexto de startups disruptivas nas práticas de inteligência competitiva - foi considerado como coberto por identificar importantes mudanças a partir do fenômeno observado. Destas mudanças, destaca-se (i) o papel da distribuição redesenhada nos meios digitais, (ii) as novas capacidades de relacionamento entre clientes e marcas de empresas nascentes, (iii) a velocidade de mudança e necessidade de amadurecimento por parte das empresas estabelecidas. Neste último elemento, a velocidade e habilidade de adaptação ao novo contexto das startups foi o diferencial em um movimento de ganho de mercado por parte da Unilever. A reflexão sobre esses três pontos traz consigo novos horizontes para a prática gerencial, bem como abre campo para futuros estudos mais aprofundados ou com métodos mais robustos. Entende-se que a fragilidade do método utilizado, uma das maiores limitações do presente estudo, pode ser compensada pela qualidade da narrativa que permeia a linha do tempo das notícias e os discursos apresentados.

No contexto da negligência levando às surpresas estratégicas, pode-se afirmar uma dupla miopia por parte da $\mathrm{P} \& \mathrm{G}$. A primeira em relação à startup emergente e a segunda em relação ao movimento de aquisição da startup por parte de sua maior rival, a Unilever. A movimentação coloca um novo tópico nas discussões sobre competitividade e inovação disruptiva: a possibilidade de pequenos entrantes virem a se tornar um elemento de disrupção de rivais mais estabelecidos por meio de aquisições, estratégias que podem se tornar cada vez mais comuns no mercado de tecnologia. Posto isso, entende-se que o presente artigo permite novos estudos no campo da inteligência competitiva, do empreendedorismo e da gestão empresarial de modo geral. Como sugestões para pesquisas futuras, uma análise quantitativa, ou estudo de casos múltiplos, pode trazer mais consistência na identificação de implicações do novo fenômeno de startups para o campo de estudos em estratégia e inteligência competitiva. 


\section{Referencial Teórico}

Alves-Mazzotti, A. J. (2006). Usos e abusos dos estudos de caso. Cadernos de pesquisa, 36(129), 637-651.

Ansoff, H. I. (1975). Managing strategic surprise by response to weak signals. California management review, 18(2), 21-33.

Ansoff, H. I. (1965). Corporate strategy: business policy for growth and expansion. McGraw-Hill Book.

Ashoka. (2016). Changemakers - Unilever Sustainable Living. Disponível em: https://www.changemakers.com/globalgoals2015\#competition-pane-1471 conteúdo.

Bardin, L. (1977). Tradução de Luis Antero Neto e Augusto Pinheiro. Análise de

Berelson, B. (1952). Content analysis in communication research.

Blanco, S., \& Lesca, H. (1998). Business intelligence: integrating knowledge into the selection of early warning signals. In Workshop on Knowledge Management.

Bower, J. L., \& Christensen, C. M. (1995). Disruptive technologies: catching the wave. Harvard Business Review, Jan-Feb, 43-53.

Caldbeck, R. (2016). Why did Unilever pay \$1B for Dollar Shave Club? TechCrunch. Disponível em: https://techcrunch.com/2016/07/22/why-did-unilever-pay-1b-for-dollar-shaveclub/

Caregnato, R. C. A., \& Mutti, R. (2006). Pesquisa qualitativa: análise de discurso versus análise de conteúdo. Texto contexto enferm, 15(4), 679-84.

Chakravorti, B. (2016). Unilever's Big Strategic Bet on the Dollar Shave Club. Harvard Business Review. Disponível em: https://hbr.org/2016/07/unilevers-big-strategic-bet-on-thedollar-shave-club

Choo, C. W. (2001). Environmental scanning as information seeking and organizational learning. Information Research, 7(1), 7-1.

Chu, B. (2015). The Black Box of Product Management. Medium. Disponível em: https://medium.com/the-black-box-of-product-management/the-black-box-of-productmanagement-3feb65db6ddb 
Christensen, C. M. (1992). Exploring the limits of the technology S-curve. Part I: component technologies. Production and Operations Management, 1(4), 334-357.

Christensen, C. M., \& Bower, J. L. (1996). Customer power, strategic investment, and the failure of leading firms. Strategic management journal, 197-218.

Christensen, C. M., Raynor, M. E., \& McDonald, R. (2015). Disruptive innovation. Harvard Business Review, 93(12), 44-53.

Gil, A. C. (2008). Métodos e técnicas de pesquisa social. 6. ed. Ediitora Atlas SA.

Govindarajan, V., \& Kopalle, P. K. (2006). The usefulness of measuring disruptiveness of innovations ex post in making ex ante predictions. Journal of product innovation management, 23(1), 12-18.

Lambert, F. (2017) Porsche changes its mind on electric vehicles, plans $50 \%$ of its production to be electric within 6 years. Electrek. Disponível em: https://electrek.co/2017/06/26/porsche-electric-vehicles-mission-e/

Lichtenthaler, E. (2003). Third generation management of technology intelligence processes. $R \& D$ Management, 33(4), 361-375.

Linanne, C. (2017). Procter \& Gamble's Gillette razor business dinged by online shave clubs. MarketWatch. Disponível em: http://www.marketwatch.com/story/procter-gamblesgillette-razor-business-dinged-by-online-shave-clubs-2017-04-26

Neff, J. (2015). Unilever Extends Foundry Program to Competitive Startups. Advertising Age. Disponível em: http://adage.com/article/cmo-strategy/unilever-extendsfoundry-program-competitive-startups/301511/

Prescott, J. E. (1995). The evolution of competitive intelligence. International Review of Strategic Management, 6, 71-90.

Schumpeter, J. (1942). Capitalism, socialism, and democracy.

Sharp, S. (2009). Competitive intelligence advantage: how to minimize risk, avoid surprises, and grow your business in a changing world. John Wiley \& Sons.

Sloan, A. P. (1964). My years with general motors. Crown Business.

Stake, R. E. (1978). The case study method in social inquiry. Educational researcher, 7(2), 5-8. 
Stratechery. (2016). Dollar Shave Club And The Disruption Of Everything. Disponível em: https://stratechery.com/2016/dollar-shave-club-and-the-disruption-of-everything/

Teo, T. S., \& Choo, W. Y. (2001). Assessing the impact of using the Internet for competitive intelligence. Information \& management, 39(1), 67-83.

Terlep, S. (2017). Gillette's Lawsuit Against Razor Startup ShaveLogic Dismissed. Fox Business. Disponível em: http://www.foxbusiness.com/features/2017/04/21/gillettes-lawsuitagainst-razor-startup-shavelogic-dismissed.html

Unilever. (2014). Unilever announces outcome of Go Global initiative. Disponível em: https://www.unilever.com/news/Press-releases/2014/14-03-13-Unilever-announces-outcomeof-Go-Global-initiative.html

Unilever. (2014). Unilever launches global platform to engage with start-ups. Disponível em: https://www.unilever.com/news/press-releases/2014/14-05-22-Unileverlaunches-global-platform-to-engage-with-start-ups.html

Unilever. (2016). Fundry - Our work. Disponível em: https://foundry.unilever.com/ourwork

Vinjamuri, D. (2012) Big Brands Should Fear The 'Dollar Shave Club' Effect. Forbes. Disponível em: https:/www.forbes.com/sites/davidvinjamuri/2012/04/12/could-your-brandbe-dollar-shave-d/\#40c6387c4854

Yin, R. K. (2013). Case study research: Design and methods. Sage publications.

Yu, D., \& Hang, C. C. (2010). A reflective review of disruptive innovation theory. International Journal of Management Reviews, 12(4), 435-452. 